\title{
Supernova physics with a low-energy beta-beam
}

\author{
Natalie Jachowicz*i \\ Department of Subatomic and Radiation Physics, Ghent University, Proeftuinstraat 86, B-9000 \\ Gent, Belgium \\ E-mail: natalie.jachowicz@UGent.be
}

\section{Gail McLaughlin}

North Carolina State University

E-mail: Gail_McLaughlin@ncsu.edu

\begin{abstract}
Core-collapse supernova neutrino-spectra are of interest not only for understanding the deep interior of astrophysical explosions, but also for understanding the synthesis of many elements made primarily in this environment. A new type of neutrino beam, the beta-beam, is now under discussion as a next generation neutrino experiment. A low-energy version of this beam has been proposed for a number of astrophysical applications. The energy range of these low-energy neutrinos is the same as that of supernova neutrinos. We present a theoretical discussion of the importance of low-energy beta-beams for improving our interpretation of a future galactic supernova signal. We present a novel method, where fitting synthetic spectra, constructed by taking linear combinations of beta-beam spectra, to the original supernova-neutrino spectra reproduces the folded differential cross sections very accurately. Comparing the response in a terrestrial detector to these synthetic responses provides a direct way to determine the main parameters of the supernovaneutrino energy-distribution.
\end{abstract}

International Symposium on Nuclear Astrophysics - Nuclei in the Cosmos - IX 25-30 June 2006

CERN

\footnotetext{
${ }^{*}$ Speaker.

${ }^{\dagger}$ Postdoctoral Fellow of the Research Foundation - Flanders (FWO)
} 

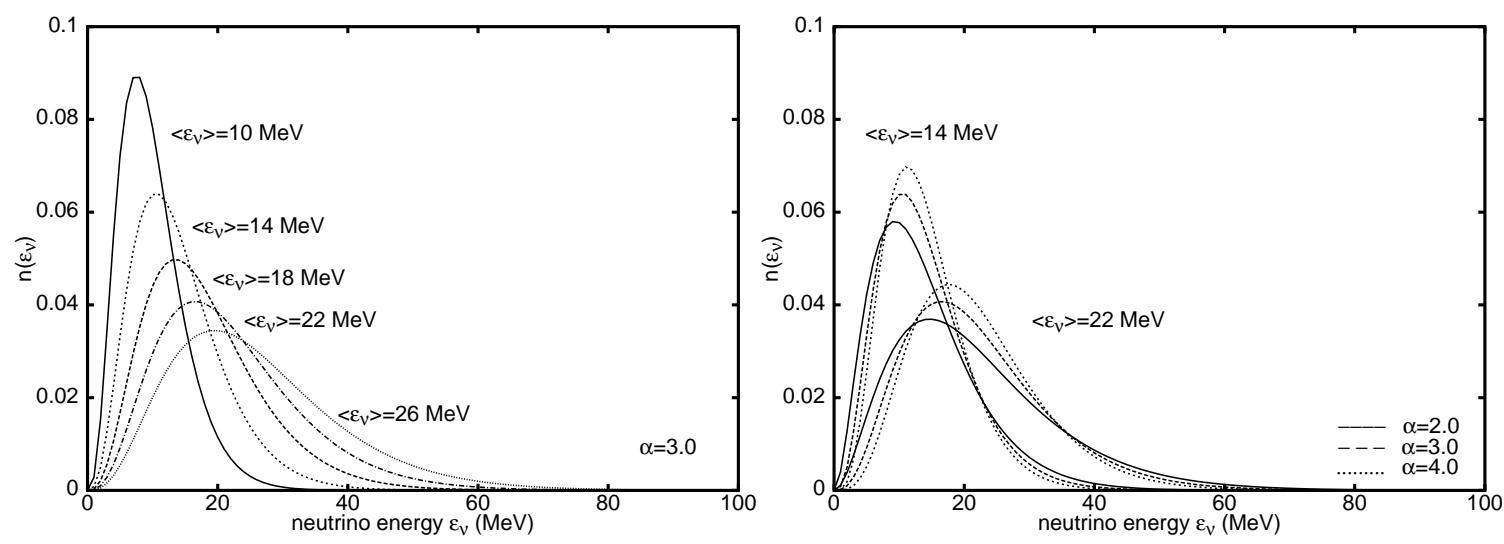

Figure 1: Power-law parametrization of the supernova-neutrino spectrum for different values of the average neutrino energy and width.

\section{Introduction}

Only weakly interacting, neutrinos are the principal messengers reaching us fro the center of a supernova. Terrestrial neutrino telescopes, such as SNO and SuperKamiokande [1,2] can provide precious information about the processes in the core of a collapsing and exploding star. But the information about the supernova that a neutrino detector can supply, is restricted by the fact that little experimental data on the neutrino-nucleus cross sections exists and by the uncertainties in theoretical calculations.

Beta beams, which are neutrino beams produced by the beta decay of nuclei that have been accelerated to high gamma factor, were original proposed for high-energy applications, such as the measurement of the third neutrino mixing angle $\theta_{13}[3]$. Volpe $[4,5,6]$ suggested that a beta beam run at lower gamma factor, would be useful for neutrino measurements in the tens of $\mathrm{MeV}$ range. The development of a beta-beam facility is currently being explored [7]. In this contribution, we investigate the interest of a low-energy beta-beam measurement for important issues in neutrino astrophysics.

\section{Construction of the synthetic responses}

Traditionally, supernova-neutrino energy-distributions were parametrized using Fermi-Dirac distributions. The spectra are however not purely thermal, as the decoupling sites of the neutrinos are influenced by their flavor and energy. Recent calculations showed that an accurate description of a supernova-neutrino spectrum are provided by a power-law distribution [8]:

$$
n_{S N[\langle\varepsilon\rangle, \alpha]}(\varepsilon)=\left(\frac{\varepsilon}{\langle\varepsilon\rangle}\right)^{\alpha} e^{-(\alpha+1) \frac{\varepsilon}{\langle\varepsilon\rangle}}
$$

where $\langle\varepsilon\rangle$ and $\alpha$ represent the average energy and the width of the spectrum respectively. The average neutrino energy $\langle\varepsilon\rangle$ is related to the temperature at the decoupling site, and the effect of $\alpha$ is equivalent to that of the introduction of the effective chemical potential in the Fermi-Dirac distribution. Neutrino-nucleus reaction cross sections depend on the square of the incoming energy, 


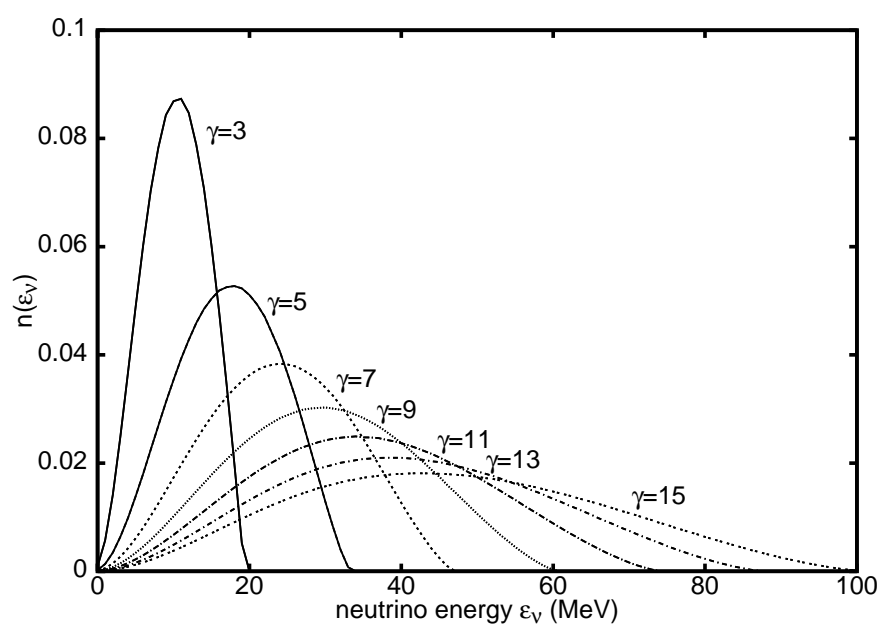

Figure 2: Beta-beam spectra stemming from ${ }^{18} \mathrm{Ne}$ decay for different values of the boost parameter $\gamma$.

thus rising very fast with neutrino energies. Hence, the folded cross sections reach their maximum at much higher energy values than the supernova-neutrino energy-spectrum does. Typically even neutrinos with energies more than twice the average energy of the distribution make sizable contributions to the folded cross section [9]. This makes the high-energy tail of the spectra very important for the determination of the nuclear response [10]. Figures 1 and 2 illustrates these distributions and compares them to beta-beam spectra at low gammas. The precise shape of the beta-beam spectra depends on the boost factor $\gamma$ of the primary ion beam and the opening angle of the flux to the target, but is remarkably similar to the supernova-neutrino energy distribution. Both classes of distributions are characterized by long tails. The range of the low-energy beta-beam spectra covers the energy region of interest for supernova neutrinos.

We exploit the flexibility offered by beta-beam facilities to construct linear combinations of beta-beam energy-distributions :

$$
n_{N \gamma}\left(\varepsilon_{i}\right)=\sum_{i=1}^{N} a_{i} n_{\gamma_{i}}\left(\varepsilon_{i}\right)
$$

where all distributions involved were normalized to 1 . The constructed spectrum that represents the best fit to the original supernova-spectrum is then obtained by minimizing the expression

$$
\int_{\varepsilon_{i}} d \varepsilon_{i}\left|n_{N \gamma}\left(\varepsilon_{i}\right)-n_{S N}\left(\varepsilon_{i}\right)\right|
$$

where the similarities between beta-beam and supernova-neutrino spectra assure that a good fit is easily obtained. In this way, the values for the expansion parameters $a_{i}$ and the boost factors $\gamma_{i}$ that yield a spectrum that is as close as possible to the original power-law distribution are determined.

Of course the spectrum as such is not an important observable. The information brought along by supernova neutrinos is encoded in the response of the detector to the incoming neutrino flux. This quantity is determined by the folded differential cross section :

$$
\sigma^{\text {fold }}(\omega)=\int_{0}^{\infty} d \varepsilon_{i} \sigma\left(\varepsilon_{i}, \omega\right) n_{S N}\left(\varepsilon_{i}\right)
$$

The folded cross section as a function of the excitation energy of the target indicates what the 

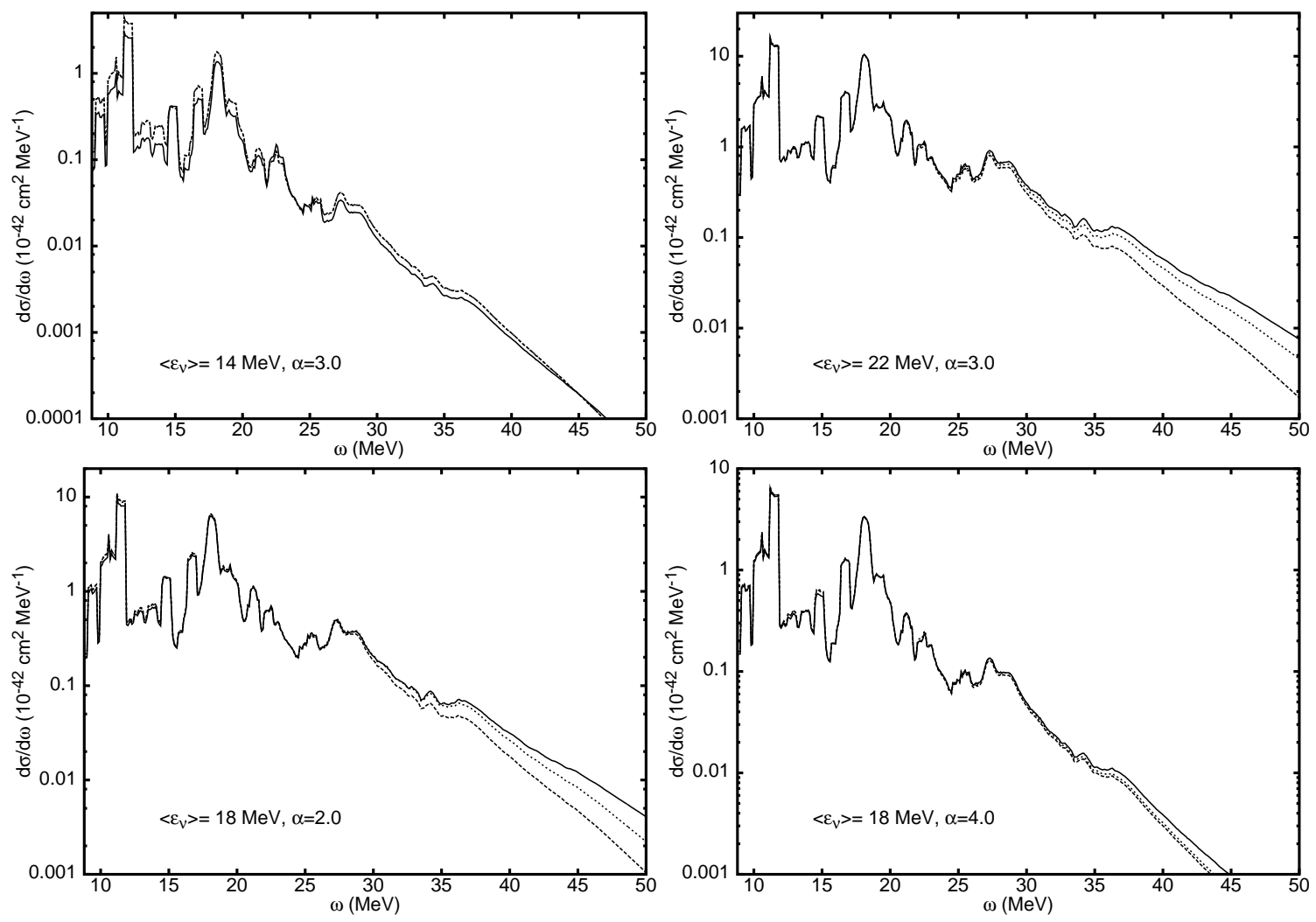

Figure 3: Comparison between differential cross sections for neutral-current scattering on ${ }^{208} \mathrm{~Pb}$, folded with a power-law supernova-neutrino spectrum (full line) and synthetic spectra with 3 (dashed line) and 5 components (dotted line) for different energy distributions. The very satisfying agreement between the differential cross setion folded with a supernova spectrum and with synthetic spectra suggests that it is possible to reconstruc and predict the supernova-neutrino signal using the results of the beta-beam measurement without going through the intermediate step of using a nuclear structure calculation. For each gamma the beta-beam response can be determined experimentally. Taking appropriate linear combinations of these responses provides a very accurate picture of the response of a terrestrial detector to an incoming supernova-neutrino signal.

neutrino signal in the detector will look like. In Figure 3, we show the differential cross section for neutral-current neutrino scattering on an ${ }^{208} \mathrm{~Pb}$ target for different energy distributions. The agreement between cross sections folded with the power-law supernova-neutrino spectrum and those folded with the synthetic spectrum is remarkably good. The procedure is able to reproduce total strength, and the position and width of the resonances very accurately.

\section{Reconstructing the supernova-neutrino spectra}

The method can be inverted to reconstruct the incoming supernova-neutrino spectrum. Given a supernova-neutrino signal and a set of beta-beam response data, fitting the supernova response in the detector to linear combinations of beta-beam responses produces the expansion parameters $a_{i}$ and $\gamma_{i}$ that yield best agreement with the supernova data. Taking the actual linear combination then reveals the original supernova-neutrino energy-distribution. This is illustrated in Fig. 4 using 


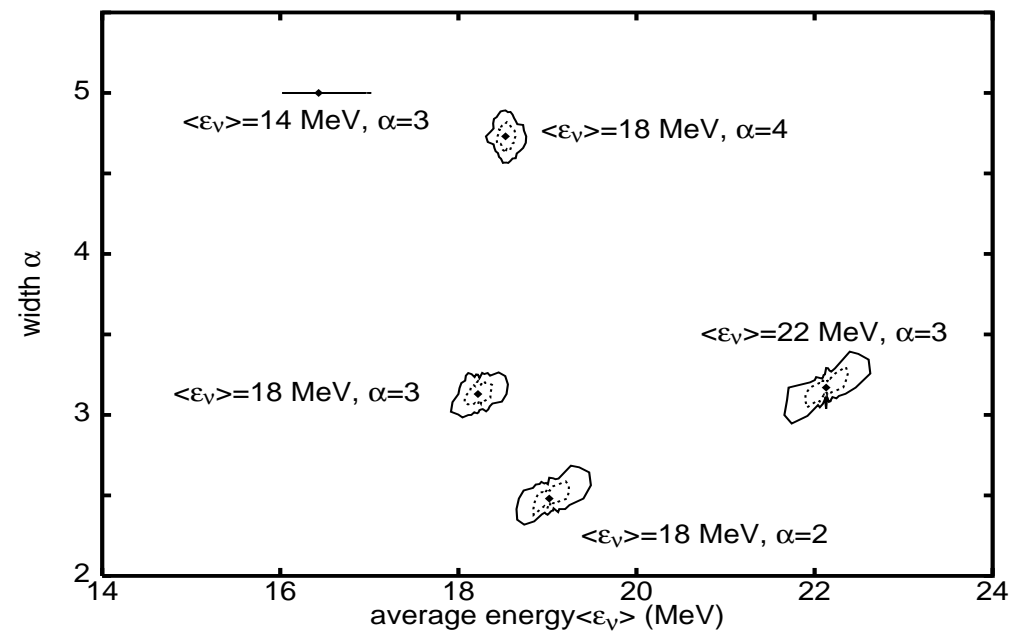

Figure 4: Reconstruction of the incident $\mathrm{SN} v$ spectrum from cross sections folded with a synthetic spectrum (dots). The dotted and full lines represent the 90\% confidence levels for constructed spectra with 5 and $10 \%$ uncertainties on the parameters $a^{\gamma_{i=1,5}}$. The mild scatter the noise produces shows that the method is stable against uncertainties at this level.

a schematic model where we create synthetic data represented by a cross section, folded with a synthetic neutrino energy distribution.

\section{Conclusion}

We propose a novel procedure [11] that determines the response of a nuclear target in a supernova-neutrino detector using data from low-energy beta-beams. We show that fitting synthetic spectra, constructed by taking linear combinations of beta-beam spectra, to the original supernovaneutrino spectra reproduces the folded differential cross sections very accurately. Comparing the response in a terrestrial detector to these synthetic responses provides a direct way to determine the main parameters of the supernova-neutrino energy-distribution.

\section{References}

[1] C.J. Clarence, the SNO Collaboration, Nucl. Phys. Proc. Suppl. 100, (2001) 326.

[2] Y. Oyama et al., Phys. Rev. Lett. 56, (1987) 2604.

[3] P. Zuchelli, Phys. Lett. B532, (2002) 166.

[4] C. Volpe, J. Phys. G30, (2004) 1.

[5] J. Serreau, C. Volpe, Phys. Rev. C 70, (2004) 055502.

[6] G.C. McLaughlin, Phys. Rev. C 70, (2004) 045804.

[7] http://beta-beam.web.cern.ch/beta-beam

[8] M. Keil, G.G. Raffelt, H.-T. Janka, Astrophys. J. 590, (2003) 971.

[9] N. Jachowicz, K. Vantournhout, J. Ryckebusch and K. Heyde, Nucl. Phys. A 758c, (2005) 51.

[10] N. Jachowicz and K. Heyde, Phys. Rev. C 68, (2003) 055502.

[11] N. Jachowicz and G.C. McLaughlin, Phys. Rev. Lett. 96, 172301 (2006). 